\title{
Féeries
}

Études sur le conte merveilleux, XVII ${ }^{\mathrm{e}} \mathrm{XIX} \mathrm{X}^{\mathrm{e}}$ siècle

Contes et morale(s)

\section{Critique et morale dans Le Taureau blanc de Voltaire (1774)}

Critique and Morality in Voltaire's Le Taureau blanc (1774)

\section{Magali Fourgnaud}

\section{(2) OpenEdition \\ 1 Journals}

Édition électronique

URL : http://journals.openedition.org/feeries/1010

ISSN : 1957-7753

Éditeur

UGA Éditions/Université Grenoble Alpes

Édition imprimée

Date de publication : 15 octobre 2016

Pagination : 183-198

ISBN : 978-2-8310-335-3

ISSN : 1766-2842

\section{Référence électronique}

Magali Fourgnaud, «Critique et morale dans Le Taureau blanc de Voltaire (1774) », Féeries [En ligne],

13 | 2016, mis en ligne le 01 janvier 2017, consulté le 08 septembre 2020. URL : http:// journals.openedition.org/feeries/1010

\section{(c) Féeries}


Magali Fourgnaud

Lycée Pape Clément (Pessac)

Université Bordeaux Montaigne

\section{CRITIQUE ET MORALE DANS LE TAUREAU BLANC DE VOLTAIRE (1774)}

$E$

N I774, VOLTAIRE, ALORS ÂGÉ DE QUATRE-VINGTS ANS, fait paraître son ultime conte oriental, Le Taureau blanc. La critique actuelle ${ }^{\mathrm{I}}$ s'accorde à lire ce récit comme une parodie des «fables ${ }^{2}$ " bibliques, que le patriarche de Ferney fustige dans l'ensemble de son œuvre. Le conte reprend d'ailleurs des arguments déjà parus dans les Questions sur l'Encyclopédie (qu'il vient d'achever lors de la parution du conte) et débattus au moins depuis les Examens de la Bible d'Émilie Du Châtelet. La verve voltairienne ne s'est pas tarie, dénonçant toujours l'immoralité et le style métaphorique des récits bibliques ${ }^{3}$, considérés comme un tissu d'invraisemblances, en opposition au "style de la raison" de Zadig. Variant les armes dans son combat contre l'Infâme, Voltaire utilise ici les procédés propres à la fiction pour démonter les croyances et les illusions qui conduisent au fanatisme : Le Taureau blanc est d'ailleurs considéré par René Pomeau comme le «seul conte de critique biblique ${ }^{4}$ » parmi la production de contes orientaux au XVIII ${ }^{\mathrm{e}}$ siècle.

I. Th. M. Mason, "A biblical conte philosophique : Voltaire's Taureau blanc", XVIIIth Century French Studies, 1968, p. 55-69; M. F. O'Meara, "Le Taureau blanc and the activity of language", SVEC, I48, 1976, p. II5-175; M.-H. Cotoni, "La Référence à la Bible dans les contes de Voltaire», dans J. Wagner (éd.), Roman et religion en France (I713-1866), Paris, Honoré Champion, 2002, p. I03-I20; M.-H. Cotoni, «Intertextualité et humour dans Le Taureau blanc de Voltaire», Cahiers de Narratologie, $\mathrm{n}^{\circ}$ 13, 2006; R. Pomeau, "Introduction", Le Taureau blanc, dans Les Euvres complètes de Voltaire, 74 A, Oxford, Voltaire Foundation, 2006, p. 3-62 (les citations renvoient à cette édition).

2. Voltaire, Article "Christianisme», Dictionnaire philosophique, R. Naves et O. Ferret (éds), Paris, Classiques Garnier, 2008, p. I26.

3. «Ezéchiel et Jérémie parlèrent aussi très longtemps dans un fort beau style qu'on pouvait à peine comprendre." Le Taureau blanc, éd. citée, p. II3.

4. R. Pomeau, art. cité, p. I5.

Féeries, ${ }^{\circ}{ }^{13}, 2016$, p. 183-198.

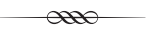

I83 
Voltaire utilise en effet ici le matériau biblique pour créer un conte de fées oriental, fantaisiste et absurde, qui se présente d'emblée comme une réécriture de plusieurs épisodes surnaturels de la Bible. Le point de départ est la métamorphose de Nabucodonosor en bœuf (Daniel, IV 22-29). Voltaire imagine le désespoir de son amante, la fille du roi de Tanis (personnage historique allié de Nabucodonosor), la princesse Amaside, dont l'éducation est confiée au sage Mambrès. Ce dernier rencontre une vieille femme, la pythonisse d'Endor (Samuel, XXVIII 7-25), accompagnée de toute une ménagerie : on reconnaît l'ânesse de Balaam (Livre des Nombres, XXII 22-24), le serpent de la Genèse, le pigeon, la colombe et le corbeau sauvés du Déluge (Genèse, III I-I6 et VIII 6-I2), le chien de Tobie (Tobie, VI I), le poisson qui avala Jonas (Jonas, II), le bouc émissaire (Lévitique, XVI 8-Io) et un taureau blanc qui n'est autre que Nabucodonosor. Touché par le désespoir de la princesse, le sage Mambrès met en place un stratagème : faire consacrer le taureau blanc comme le nouveau dieu Apis (l'ancien venant justement de mourir). Mambrès utilise les animaux bibliques comme coursiers pour prévenir le prêtre de Memphis. Sur ces entre-faits, arrivent les trois prophètes, Daniel, Ezéchiel et Jérémie, qui, après un repas gargantuesque, se trouvent métamorphosés en pies par «le maître du monde». Puis pour distraire la princesse de son sort, le serpent développe trois histoires (un récit mythique, un conte libertin et un conte moral), qui ennuient profondément Amaside. Alors que la belle et le bœuf s'apprêtent, la première, à avoir le cou coupé pour avoir prononcé le nom de son amant, le second, à être brûlé pour sorcellerie, une foule innombrable arrive pour célébrer l'avènement du nouveau dieu. C'est à ce moment-là que les sept années de malédiction prennent fin : Nabucodonosor retrouve forme humaine, épouse la princesse et devient un roi puissant. Désormais, à Babylone, à chaque fois qu'un souverain reconnait ses erreurs et les corrige, on s'écrie : "Vive notre grand roi qui n'est plus bœuf ${ }^{5}$ ! Cette transposition du merveilleux chrétien au cœur de la féerie vide l'Écriture de toute dimension religieuse, la réduisant à une histoire bouffonne, légèrement érotique. En ce sens, le conte suit la même démarche critique que le Dictionnaire philosophique. À la fin de l'article «Ezéchiel (d')», par exemple, au grave savant qui considère les viols et les incestes rapportés par l'Ancien Testament comme des «fictions ingénieuses et toutes remplies d'agrément", un jeune homme fort instruit répond : «[...] si vous voulez des fictions, croyez-moi, préférez celles d'Homère,

5. Le Taureau blanc, éd. citée, p. 135. 
de Virgile, d'Ovide ${ }^{6}$.» Dès lors, en quoi la critique par le conte diffèret-elle de celle du commentaire? En quoi est-elle complémentaire de la démarche analytique du Dictionnaire et de l'Encyclopédie?

Le Taureau blanc se caractérise tout d'abord par la pauvreté de sa trame narrative. Loin des longues pérégrinations de Candide, de Scarmatendo ou de Jenni à travers le monde et à l'opposé des facétieuses péripéties de Zadig ou de Memnon, l'action est ici réduite à portion congrue, l'intérêt du conte résidant avant tout dans la virulence de sa critique à l'égard de la Bible. Alors que, pour tenter de consoler la princesse Amaside, le serpent lui raconte des épisodes bibliques célèbres, la belle, fatiguée de cette énumération de massacres, d'incestes et de viols ${ }^{7}$, s'écrie : "Tous ces contes-là m'ennuient [...]. Ils ne sont bons que pour être commentés chez les Irlandais par ce fou d'Abadie, ou chez les Welches par ce phrasier d'Houteville ${ }^{8}$.» Outre l'effet comique de la mention, au cœur de la fiction orientale, de deux personnages réels, adversaires habituels de la polémique voltairienne, l'attaque directe de ces deux apologistes révèle la véritable cible de la critique, à savoir l'exégèse biblique, dont les interprétations erronées conduisent aux pires atrocités :

Tous les ministres d'État conclurent que le taureau blanc était un sorcier. C'était tout le contraire, il était ensorcelé; mais on se trompe toujours à la cour dans ces affaires délicates. On conclut à la pluralité des voix qưil fallait exorciser la princesse, et sacrifier le taureau blanc et la vieille?

Le passage n'est pas sans évoquer le chapitre vi de Candide mettant en scène l'autodafé auquel sont condamnés le jeune naïf et son mentor, «l'un pour avoir parlé, l'autre pour avoir écouté». Si la dénonciation semble moins spectaculaire dans Le Taureau blanc que dans Candide, Zadig ou L'Ingénu, c'est que l'attaque vise ici l'Écriture.

En effet, l'ironie voltairienne s'en prend non seulement aux prophètes ${ }^{10}$ (Mambrès et Moïse sont, par antiphrase, de "profonds philosophes ${ }^{\mathrm{II}} »$ ),

6. Voltaire, Dictionnaire philosophique, ouvr. cité, p. I89.

7. "Il lui racontait les amours malheureux de Sichem et de l'agréable Dina âgée de six ans, et les amours plus fortunés de Booz et de Ruth, ceux de Juda avec sa bru Thamar, ceux de Loth avec ses deux filles qui ne voulaient pas que le monde finît, ceux d'Abraham et de Jacob avec leurs servantes, ceux de Ruben avec sa mère, ceux de David et de Bethsabée, ceux du grand roi Salomon [...].» Le Taureau blanc, éd. citée, p. II9-I2O.

8. Ibid., p. I2I.

9. Ibid., p. 9I.

Io. "Cependant le métier de prophète a du bon; et la preuve en est que mille gens s'en mêlent." Ibid., p. II2.

II. Ibid., p. 65. 
mais surtout à leurs prédictions, assimilés au caquet des pies $^{12}$. Les malédictions de la vieille pythonisse, qui cherche à persuader le taureau de ne pas chercher à "rompre sa chaîne», sont également tournées en dérision : "Ce gros poisson que vous voyez, l'avalerait infailliblement, et le garderait plus de trois jours dans son ventre; ou bien ce serpent qui vous a paru peut-être assez doux et assez aimable, lui pourrait faire une piqûre mortelle $^{\mathrm{r}}$.» Les amplifications et les tournures hyperboliques désacralisent la parole religieuse : par le pastiche, Voltaire utilise les outils de ses ennemis, à savoir le recours aux images fortes, capables de frapper les esprits et de jouer sur les peurs naïves ${ }^{14}$, pour mieux en montrer l'artificialité et en dénoncer les effets. La pythonisse d'Endor berce de doux contes ceux qui croient, mais le conte les réveille en supprimant tout recours au merveilleux : les ombres nocturnes qu'elle fait apparaitre au roi Amasis n'ont aucun pouvoir sur lui. L'Écriture est alors vidée de tout pouvoir référentiel ou performatif et se trouve même assimilée à une rumeur plus ou moins fiable :

On dit même qu'il fut chassé autrefois d'un beau lieu pour son excès d'orgueil. — Je ne l'ai jamais ouï dire, repartit la princesse. - Je le crois bien, reprit le vieillard. Alors il lui apprit tous les bruits qui avaient couru sur ce serpent si fameux ${ }^{15}$.

Rapportés, allongés, modifiés, les récits bibliques sont réduits à des commérages, à l'image des ragots déployés par les dames de compagnie ${ }^{16}$. Dès l'incipit, le narrateur fait allusion à la dispute «fameuse», qui eut lieu entre le sage Mambrès et Moïse, "comme on sait ${ }^{17}$ ». Ainsi, Voltaire "ramèn[e] le merveilleux chrétien à l'universelle superstition ${ }^{18}$ » et il rejoint, en ce

I2. Les trois prophètes, Daniel, Ezéchiel et Jérémie, transformés en pie, "continuèrent de parler comme auparavant", ibid., p. II3.

13. Ibid., p. 76.

14. Exemple d'exercices spirituels imposés aux novices par les Jésuites : «Lorsqu'il médite sur l'enfer, il doit voir une plaine enflammée, des âmes brûlées dans des corps de feu, entendre des hurlements, des blasphèmes; [...] Présenter ces exercices à de jeunes gens d'une imagination vive et forte, comme des voies ordinaires de perfection; les proposer dans la vie commune à des hommes, à des femmes, comme elles sont proposées et vantées dans les constitutions, c'est inspirer l'enthousiasme et préparer les voies au fanatisme.» L.-R. De Caradeuc de la Chalotais, Compte rendu des constitutions des Jésuites, [Conclusions devant le Parlement de Rennes, 7 décembre 1761], I762, p. 173-175. Je remercie Florence Boulerie de m'avoir fait découvrir ce texte.

15. Le Taureau blanc, éd. citée, p. 80.

16. Ibid., p. 90.

17. Ibid., p. 65 .

I8. M.-H. Cotoni, art. cité, 2002, p. I09. 
sens, le Diderot des Bijoux indiscrets ${ }^{19}$. Mais la parodie biblique va plus loin que la simple satire des croyances : le conte voltairien remet explicitement en question la portée même de la Bible.

Le narrateur voltairien, tout en établissant une connivence culturelle avec le lecteur, insiste, de manière paradoxale et comique, sur l'insignifiance de personnages aussi célèbres que le serpent, Noé, cet «inconnu à toutes les nations ${ }^{20}$ ", ou même Ėve, "notre mère commune dont [la princesse a] oublié le nom ${ }^{2 \mathrm{I}} »$. Ces précisions, par leurs effets burlesques, désacralisent l'Écriture, relativisent son importance et tournent en dérision sa prétention à l'universalité et à la vérité. Les récits bibliques n'ont d'ailleurs qu'une valeur culturelle et leur rayonnement est circonscrit à un espace limité : «[...] l'Éternel révèle ce qu'il veut et à qui il veut, illustre pythonisse. Toutes ces bêtes qui sont commises avec vous à la garde du taureau blanc, ne sont connues que de votre généreuse et agréable nation, qui est elle-même inconnue à presque tout le monde ${ }^{22}$.» De nouveau le conte rejoint la démarche du Dictionnaire philosophique. L'article "Abraham», par exemple, s'ouvre également sur une dépersonnalisation du patriarche ${ }^{23}$. Il s'agit dans les deux cas de démonter la suprématie symbolique d'Israël, domination que Voltaire raille dans l'article «Juifs» des Questions sur l'Encyclopédie $^{24}$. Face aux grandes puissances que sont l'Inde, l'Égypte, ou la Perse, que peut bien représenter «ce pays affreux [qui] n'a pas plus de huit lieues de long et de large sur toutes les $\operatorname{cartes}^{25}$ », et que l'on considère pourtant comme le berceau de l'humanité?

Le conte oriental relativise ainsi la portée de la Bible, qu'il ramène à sa dimension littéraire. À l'instar du Dictionnaire philosophique qui invite

19. "La religion revendiqua leur caquet comme une matière de sa compétence, et ses ministres prétendirent que le doigt de Brama se manifestait dans cette œuvre.» D. Diderot, Les Bijoux indiscrets, dans M. Delon (éd.), Contes et romans, Paris, Gallimard, coll. "Bibliothèque de la Pléiade», 2004, p. 40.

20. Le Taureau blanc, éd. citée, p. I04.

2I. Ibid., p. 82.

22. Ibid., p. 74-75.

23. "Abraham est un de ces noms célèbres dans l'Asie Mineure et dans l'Arabie, comme Thaut chez les Égyptiens, le premier Zoroastre dans la Perse, Hercule en Grèce, Orphée dans la Thrace, Odin chez les nations septentrionales, et tant d'autres plus connus par leur célébrité que par une histoire très avérée." Dictionnaire philosophique, ouvr. cité, p. 6.

24. «Ainsi après les Chaldéens, les Syriens, les Perses, les Phéniciens, les Égyptiens, les Grecs, les Indiens, les Chinois, les Latins, les Toscans, vous êtes le premier peuple de la terre qui ait eu quelque forme de gouvernement connu.» Article "Juifs», Questions sur l'Encyclopédie, dans CEuvres complètes de Voltaire, 42 A, C. Mervaud et N. Cronk (éds), Oxford, Voltaire Foundation, 2OII, p. $47 \mathrm{I}$.

25. Ibid., p. 485 . 
à ne lire l'histoire de Joseph que «comme un objet de curiosité et de littérature $^{26}$ ", Le Taureau blanc rapproche sans cesse les épisodes bibliques surnaturels des Métamorphoses d'Ovide : l'Ancien et le Nouveau Testaments n'ont pas plus d'importance (voire moins) que les mythes antiques. L'Écriture apparaît non comme une parole sacrée, mais comme une source inépuisable pour la création, comme l'illustre le renversement carnavalesque de la Genèse et du Déluge. Le corbeau explique, par exemple, comment Noé et sa femme, "qui n'avaient guère plus de six cents ans ${ }^{27}$ ", s'étaient mis entièrement au service des animaux, leur préparant des repas gargantuesques; ailleurs, le serpent tentateur responsable de la Chute se trouve flatté par Amaside: «[...] il est juste qu’à son tour une femme le séduise $^{28}$.» Ironiquement, le serpent est même présenté comme un chantre des Lumières (un avatar de Voltaire?), défenseur de la connaissance et de la curiosité : "Le maître aurait-il voulu être servi par des ignorants et des idiots? L'esprit n'est-il pas fait pour s'éclairer, pour se perfectionner? $\mathrm{Ne}$ faut-il pas connaître le bien et le mal pour faire l'un et pour éviter l'autre? Certainement on me devait des remerciements ${ }^{29}$.» Par le recours au burlesque, Voltaire tourne en dérision l'usage religieux des symboles, car il est faux et dangereux. Ces réécritures témoignent également de la réversibilité de l'interprétation de ces motifs : placés dans un cadre narratif différent, on peut leur accorder des significations totalement opposées.

En ce sens, le conte voltairien propose un dispositif cognitif qui s'apparente aux "ruptures de cadrages», tels que les décrit le sociologue Erwin Goffman. Ce dernier définit la notion de «cadre» comme «ce qui nous permet, dans une situation donnée, d'accorder du sens à tel ou tel de ses aspects, lequel autrement serait dépourvu de significations ${ }^{30} »$. Le cadre structure les événements et en oriente notre perception et donc notre interprétation. Dès lors, les ruptures (par fabrications ou modélisations) ont pour effet d'une part de nous déstabiliser, d'autre part de nous rendre conscients du cadre et de sa relativité. Dans le conte voltairien, l'insertion des personnages bibliques dans des cadres narratifs différents conduit le lecteur à porter un nouveau regard sur ce qu'il connaît, et à considérer ces motifs dans leur dimension littéraire et non sacrée. En effet, la première

26. Article "Joseph», Dictionnaire philosophique, ouvr. cité, p. 249.

27. Le Taureau blanc, ouvr. cité, p. IO4.

28. Ibid., p. 8o.

29. Ibid., p. 83 .

30. E. Goffman, Les Cadres de l'expérience [1974], trad. I. Joseph, M. Dartevelle et P. Joseph, Paris, Éditions de Minuit, I99I, p. 30. 
fois qu'ils apparaissent, la vieille pythonisse ${ }^{31}$ et le bestiaire qui l'accompagne semblent tout droit sortis d'un conte traditionnel ou d'une fable animalière :

Comme elle avançait en silence vers les bords du Nil, elle aperçut de loin sous un bocage baigné par le fleuve, une vieille femme couverte de lambeaux gris, assise sur un tertre. Elle avait auprès d'elle une ânesse, un chien, un bouc. Vis-à-vis d'elle était un serpent qui n'était pas comme les serpents ordinaires, car ses yeux étaient aussi tendres qu’animés; sa physionomie était noble et intéressante; sa peau brillait des couleurs les plus vives et les plus douces. Un énorme poisson à moitié plongé dans le fleuve, n'était pas la moins étonnante personne de la compagnie. Il y avait sur une branche un corbeau et un pigeon. Toutes ces créatures semblaient avoir ensemble une conversation assez animée $^{32}$.

Quelques pages plus loin, les mêmes personnages se trouvent placés dans un environnement bucolique et romanesque: "La dame d'honneur causa avec l'ânesse. Les dames de compagnie s'amusèrent avec le bouc, le chien, le corbeau et la colombe ${ }^{33}$.» Le décor pastoral ("sous un bocage») est alors propice à l'insertion d'un dialogue théâtralisé entre la princesse et le serpent, dont le marivaudage est émaillé de termes précieux («sous le ciel empyré»). Ces changements de cadres génériques modifient totalement leur interprétation : le chien de Tobie et le bouc émissaire deviennent, de manière comique, de simples animaux de compagnie et le serpent tentateur se présente comme "un favori disgracié». Ces renversements structurent véritablement l'ensemble du conte : ils constituent à la fois la matière du récit et le cadre du dispositif expérimental car ils sont les conditions nécessaires à l'émergence d'une vérité morale. En effet, loin de tout relativisme radical, Voltaire pousse son lecteur, grâce à la multiplication des significations possibles, à faire usage de sa raison, en somme il l'engage dans sa recherche de la vérité. Daniel, Ezéchiel et Jérémie sont ainsi présentés non comme des prophètes mais comme des personnages romanesques : "[...] trois graves personnages, à demi vêtus de lambeaux crasseux et déchirés, mais conservant, sous ces livrées de la pauvreté, l'air le plus majestueux et le plus auguste ${ }^{34}$. " Certes ces portraits parodiques désacralisent l'Écriture, mais ces métamorphoses ont également une fonction heuristique : grâce à ces réécritures multiples, les personnages, dont

31. René Pomeau signale qu'avant Le Taureau blanc, elle était déjà devenue un personnage de fiction dans l'œuvre de Voltaire, notamment sous les traits d'une sorcière dans le chant IV de $L a$ Pucelle, ouvr. cité, p. $72, \mathrm{n}^{\circ} 3$.

32. Le Taureau blanc, éd. citée, p. 67.

33. Ibid., p. 8I.

34. Ibid., p. 107. 
l'apparence varie en fonction des cadres génériques dans lesquels ils s'insèrent, échappent à toute fermeture interprétative. Plus précisément, ils se chargent d'une épaisseur de significations hétérogènes. Mambrès établit lui-même plusieurs hypothèses pour reconnaître le serpent qui accompagne la vieille pythonisse : il y voit tour à tour le symbole de l'éternité chez les Égyptiens, l'emblème du dieu Esculape, une des métamorphoses de Jupiter et conclut de cette énumération que «tous ces serpents-là sont de la même famille ${ }^{35}$ ». De fait, ces multiples lectures mettent en évidence la dimension symbolique de l'animal. En soulevant ce questionnement sur les signes et sur leurs significations, le conte oriental rejoint à nouveau la démarche du Dictionnaire philosophique. Dans l'article "Apis», Voltaire fustigeait déjà la confusion entre le mot et la chose : «Le bœuf Apis était-il adoré à Memphis comme dieu, comme symbole, ou comme bœuf? Il est à croire que les fanatiques voyaient en lui un dieu, les sages un simple symbole, et que le sot peuple adorait le bøuf ${ }^{36}$.» Dans le conte, le prophète Ezéchiel rappelle lui-même que Oolla et Ooliba sont des «fleurs de rhétorique $^{37}$ ». La lutte contre l'Infâme passe donc par une réflexion d'ordre sémiologique, pour que l'on cesse de confondre le symbole et son référent. Dans le conte, le recours quasi systématique au double sens participe également à cette démarche : le taureau blanc «ne pouvait parler», "mais toutes ses actions étaient éloquentes »; il est considéré par la vieille pythonisse comme un "animal impayable ${ }^{38}$. Ces jeux de mots humoristiques font miroiter le sens, poussant le lecteur à chercher systématiquement un sens figuré. Or Voltaire joue avec les différentes strates de signification et c'est finalement l'emploi du sens propre qui crée un effet comique en tournant en dérision l'absurdité de l'interprétation religieuse : pour sauver le bœuf, on ne peut même pas le mettre dans une écurie ${ }^{39}$. Les réécritures bibliques participent donc au projet critique de Voltaire et à sa volonté de transmettre l'esprit philosophique : le conte oriental offre au lecteur une expérience du renversement qui vise à remettre en question l'interprétation religieuse des symboles et l'autorité de la Bible. Mais rejetant avec ferveur l'esprit de sérieux et toute fermeture dogmatique du sens, le conte poursuit le retournement jusqu'au bout en se prenant lui-même comme cible de la critique.

35. Ibid., p. 79.

36. Article "Apis", Dictionnaire philosophique, ouvr. cité, p. 3 I.

37. Le Taureau blanc, éd. citée, p. II2.

38. Ibid., p. 69.

39. Ibid., p. 97. 
La construction emboîtée est certes une reprise de la manière des Mille et Une Nuits et la technique est utilisée depuis une longue tradition européenne $^{40}$. Mais chez Voltaire, comme d'ailleurs chez Diderot et Crébillon, elle prend une visée cognitive : la présentation du conte comme une traduction ${ }^{41}$, le découpage en chapitres et les nombreuses expressions métafictionnelles rendent le lecteur sans cesse conscient des ficelles narratives. Le serpent par exemple revendique son ontologie fictionnelle : "Les bracmanes sont les premiers qui ont donné une longue histoire de mes aventures. Je ne doute pas que des poètes du Nord n'en fassent un jour un poème épique bien bizarre; car en vérité c'est tout ce qu'on en peut faire ${ }^{42}$.» Le personnage biblique est ainsi ramené à sa fonction littéraire, chaque conteur se l'appropriant d'un bout à l'autre de la planète. La vieille pythonisse remarque également avec humour la coïncidence hasardeuse de sa rencontre avec le sage Mambrès, assimilant les pouvoirs du conteur à ceux d'un démiurge : "Ce n'est pas sans doute sans un ordre de la Providence universelle que nous nous retrouvons dans cette prairie sur les rivages du Nil, près de la superbe ville de Tanis ${ }^{43}$. " À la manière des contes orientaux de Crébillon et de Diderot, Le Taureau blanc devient un laboratoire des fictions et de leurs interprétations.

Dans les chapitres VIII et IX en particulier, sont insérés les contes que le serpent déploie afin de consoler la princesse. Les interventions de la jeune auditrice qui coupe son conteur ${ }^{44}$ ne sont pas sans évoquer les «réflexions» du sultan dans Le Sopha ou les commentaires de la sultane dans le conte de Diderot, L'Oiseau blanc, conte bleu. La réponse du serpent ( Prenez garde, si vous n'avez pas plus de pouvoir sur vous-même, vous êtes perdue.») résonne pour sa part comme un pastiche du Magasin des enfants de Madame Leprince de Beaumont et des dialogues pédagogiques enchâssés entre les contes ${ }^{45}$. Le serpent se lance tout d'abord dans la reprise du mythe antique d'Amphion, architecte de Thèbes, mêlant à son tour les références bibliques à la mythologie grecque : «[...] un rigodon

40. J.-Fr. Perrin, L'Orientale allégorie. Le conte oriental au XVIII' siècle en France (I704- I774), Paris, Honoré Champion, 2015, p. I03.

4I. Le sous-titre précise que le conte est «traduit du syriaque par M. Mamaki, interprète du roi d'Angleterre pour les langues orientales». Un sous-titre d'une version précédente attribue la parenté de l'histoire à Dom Calmet, l'auteur du Commentaire littéral sur tous les livres de l'Ancien et du Nouveau Testament. Le Taureau blanc, éd. citée, p. 63.

42. Ibid., p. 8I-82.

43. Ibid., p. 72.

44. «Un jeune roi! beau, fait à peindre, amoureux, aimé! et de qui? et quel était ce roi? quel âge avait-il ? qu'est-il devenu? où est-il? où est son royaume? quel est son nom?» Ibid., p. 86.

45. J.-Fr. Perrin, ouvr. cité, p. 93. 
et un menuet lui suffisaient pour bâtir une ville, mais l'autre les détruisait au son du cornet à bouquin $[. . .]^{46}$." Après l'échec des récits bibliques qui ennuient son auditrice, le serpent se lance dans un conte libertin : l'histoire du roi Gnaof ${ }^{47}$ et de la reine Patra, dans lequel on peut repérer des allusions au conte philosophique de Rousseau, La Reine Fantasque ${ }^{48}$. Toujours insatisfaite, la princesse veut une "fable bien vraie, bien avérée et bien morale", capable de lui "former l'esprit et le cœur " ${ }^{49}$. S'ensuit l'histoire des trois prophètes qui, alors qu'ils sont condamnés à satisfaire éternellement leurs passions (la rhétorique pour l'un, la musique pour l'autre et les femmes pour le dernier), restent mécontents de leur sort. Cet apologue, dont la morale implicite est «Toujours du plaisir n'est pas du plaisir", est construit sur le modèle des contes didactiques que Fénelon a écrits pour l'éducation du duc de Bourgogne ${ }^{50}$. Ces trois récits représentent les différentes modalités du conte à visée morale au XVIII ${ }^{\mathrm{e}}$ siècle, du conte didactique au conte libertin ${ }^{S I}$ : comme l'affirme Jean-François Perrin, qui a montré ce que Voltaire doit également à Hamilton, le serpent décline en une page "une sorte d'anthologie parodique des principales manières en $\operatorname{cours}^{52}$ ». Outre la grande connaissance du genre dont elles témoignent, ces insertions métafictionnelles ont une fonction heuristique car elles permettent au lecteur d'observer à la fois le mécanisme de création des histoires instructives et leur réception.

Paradoxalement, alors qu'elle réclame avec vigueur des récits édifiants, la princesse semble ne pas comprendre la portée morale du dernier conte : " [...] je sais bien que je ne demanderais rien à personne, si j'étais enfermée tête à tête avec mon amant, avec mon cher Nabucodonosor ${ }^{53}$." Sa réponse, outre l'allusion grivoise et comique, suggère qu'elle n'a pas perçu l'exhortation à la patience et l'invitation à accepter justement la limitation de ses passions. Le conte met ainsi en scène une double réception du conte emboîté, celle de la princesse et celle du lecteur, conduisant ce dernier à expérimenter la pluralité des interprétations possibles. Après chaque aven-

46. «Le son du cornet à bouquin» est une allusion comique au son des trompettes capables de détruire Jericho (Josué). Le Taureau blanc, éd. citée, p. II7.

47. Serait-ce une anagramme de Fagon, le médecin de Louis XIV?

48. J.-Fr. Perrin, ouvr. cité, p. 92-93.

49. Le Taureau blanc, éd. citée, p. I24.

50. Fénelon, Fables et opuscules pédagogiques, dans CEuvres I, J. Le Brun (éd.), Paris, Gallimard, coll. «Bibliothèque de la Pléiade», I983.

5I. Le premier opus sous-titré "conte moral» est le récit libertin de Crébillon, Le Sopha, conte moral (1742).

52. J.-Fr. Perrin, ouvr. cité, p. 87.

53. Le Taureau blanc, éd. citée, p. 127. 
ture, Amaside et son mentor opèrent un retour sur leur propre situation et se plongent dans des "réflexions" ou de "sérieuses pensées " ${ }^{4}$. Mambrès est d'ailleurs appelé, avec une épithète homérique, "le réfléchissant ${ }^{55}$ ". Là encore cette alternance entre action et commentaires rappelle la construction des contes crébilloniens, Tanzaï et Néardané ou Le Sopha: dans le premier, Tanzaï reproche à la fée Moustache son "maussade jargon ${ }^{56}$; ; dans le second, le sultan fustige les "chiennes de réflexions» et il est même prêt à tuer "le premier qui osera [lui] faire une réflexion ${ }^{57}$. Ces coupures de la narration soulignent avec humour l'autoréflexivité du conte qui, en parodiant sa propre réception, rejette toute lecture dogmatique. Dans Le Taureau blanc, les insistants effets d'analogie entre l'histoire rapportée et la situation de l'auditeur, technique narrative déjà éculée, prennent également des accents parodiques. Amaside compare ainsi, sur un mode comique, l'état de la pythonisse au sien : "La princesse songeant que le serpent avait appelé la vieille mademoiselle, conclut au hasard qu'elle était pucelle, et sentit quelque affliction de l'être encore $[\ldots]^{58}$.» Le sage Mambrès, quant à lui, interprète chaque aventure et cherche à lui donner un sens moral : "Voilà, disait-il, trois grands prophètes changés en pies; cela doit nous apprendre à ne pas trop parler, et à garder toujours une discrétion convenable. Il concluait que sagesse vaut mieux qu'éloquence, et pensait profondément selon sa coutume, lorsqu'un grand et terrible spectacle vint frapper ses regards $s^{59}$." Le retour de la narration au cœur même de la réflexion du mentor vient ironiquement bousculer sa prétention à la sagesse. L'humour du narrateur prend donc pour cible les oeillères et la lecture égocentrique de ses propres personnages, qui ne lisent ce qui leur arrive ou ce qui leur est raconté qu'en fonction de leur expérience. Un voile parodique s'applique ainsi sur l'ensemble de la fiction, faisant vaciller sa propre présomption à soulager ses lecteurs et à transmettre des valeurs morales. Les questions que soulèvent la princesse («[...] les exemples consolent-ils? Si mon amant était mort, me consolerais-je par l'idée que tous les hommes meurent ${ }^{60}$ ?») sont si convenues qu'elles laissent entendre

54. Ibid., p. 69.

55. Ibid., p. 154.

56. C.-P. Joliot de Crébillon, Tanzaï et Néardané, histoire japonaise, dans Euvres complètes, t. I,

J. Sgard (éd.), Paris, Classiques Garnier, 1999, p. 352.

57. C.-P. Joliot de Crébillon, Le Sopha, conte moral, Paris, Garnier Flammarion, I995, p. 50.

58. Le Taureau blanc, éd. citée, p. 77.

59. Ibid., p. II3.

6o. Ibid., p. 96. 
une «ironie désenchantée ${ }^{6 \pi}$ » à l'égard des pouvoirs du conte, autodérision qui se laisse percevoir également dans le long développement métapoétique d'Amaside :

Je veux qu'un conte soit fondé sur la vraisemblance, et qu'il ne ressemble pas toujours à un rêve. Je désire qu'il n'ait rien de trivial ni d'extravagant. Je voudrais surtout que, sous le voile de la fable, il laissât entrevoir aux yeux exercés quelque vérité fine qui échappe au vulgaire ${ }^{62}$.

Autour des années 1770, Voltaire s'oriente effectivement vers une esthétique plus réaliste, notamment dans l'Histoire de Jenni ou le Sage et l'Athée (parue la même année que Le Taureau blanc), courant lui-même le risque de rater sa cible, comme en témoigne ce commentaire des Mémoires secrets: «En général, cet ouvrage n'approche pas des autres opuscules de l'auteur en ce genre, où le raisonnement est mis en action et a plus de force ${ }^{63}$.» Paradoxalement, cette surenchère laudative du conte moral (sur le modèle de ceux de Marmontel) dans la bouche de la princesse, lectrice de Locke et de La Fontaine, évoque incontestablement l'introduction du "conte moral» de Crébillon, Le Sopha, dont le narrateur soutient ironiquement que « [l] es importantes leçons que les Contes renferment [...] ne prennent rien sur le vulgaire ${ }^{64} »$. Comme chez son prédécesseur, les contradictions du conte voltairien laissent planer de l'ironie sur l'éloge excessif des histoires instructives. Bien étrange apologie d'un conte vraisemblable qui "ne ressemble pas toujours à un rêve» à l'intérieur même d'un récit dont le fil narratif enchaîne les événements sans logique apparente ${ }^{65}$, comme par association d'idées ${ }^{66}$. Les effets de polyphonie mettent donc en scène une double interprétation des récits enchâssés, l'une dite "morale", c'està-dire instructive, et l'autre sceptique et désenchantée. Cette superposition des lectures confere au conte une portée philosophique, et ce pour deux raisons : l'insertion de connotations antinomiques perturbe la per-

6I. J.-Fr. Perrin, ouvr. cité, p. 87.

62. Ibid., p. I22.

63. Cité par R. Démoris, "Introduction", Histoire de Jenni ou le Sage et l'Athée, dans Euvres complètes, 76, N. Cronk (éd.), Oxford, Voltaire Foundation, 2013, p. 3; et par C. Duflo, "Comment rater un conte philosophique?», Cahiers Voltaire, n I4, Ferney, 2015, p. 9.

64. C.-P. Joliot de Crébillon, Le Sopha, éd. citée, p. 30.

65. Le conte présente une chronologie et une géographie fantaisistes : on passe par Canope pour aller à Tanis et Hiram est considéré comme le contemporain du pharaon Nephel Kerès ou de la guerre de Troie; Mambrès, quant à lui, écrit une lettre au grand prêtre de Memphis sur du papyrus, "qui n'était pas encore d'usage». Le Taureau blanc, éd. citée, p. 7I et Ioo.

66. Par exemple: «Tandis qu'il était plongé dans cette métaphysique obscure, comme l'est toute métaphysique un batelier, en chantant une chanson à boire, amarra un petit bateau près de la rive.» Ibid., p. I07. 
ception du lecteur, participe à la remise en question de ses préjugés et le pousse à penser par lui-même; mais surtout, la philosophie voltairienne s'investit ici dans cette esthétique des contraires.

En effet, le conte tisse tout un réseau d'analogies entre des éléments en apparence contradictoires. Le serpent, par exemple, compare sa situation à celles des ministres, savants et autres "grands génies [...] persécutés pour avoir écrit des choses utiles au genre humain ${ }^{67}$ ». La parodie satirique s'entend également comme une allusion à l'exil forcé de Voltaire lui-même. Les annotations en bas de page (notamment sur les équivalences monétaires) établissent des liens entre l'histoire et le contexte culturel du lecteur. Les comparaisons émaillent d'ailleurs l'ensemble du texte : la colère de Tanis est rapprochée de celle de Minos, l'histoire de Noé de celle du roi de Thrace Xissutre, la transformation des prophètes en pies de celles des Pierides, Moïse est assimilé à Bacchus. Ces effets d'analogie permettent à Voltaire de recréer «une sorte de poétique de la contiguïté universelle, où dieux et hommes, humains et animaux, plantes et minéraux participent d'une même harmonie ${ }^{68}$ " : «rerum concordia discors ${ }^{69}$ ", déclame Mambrès, plagiant son maitre Zoroastre. Or ce rapprochement des contraires est l'expression esthétique de la philosophie voltairienne qui cherche à mettre au jour les contradictions humaines ${ }^{70}$ pour mieux les accepter : "Et s'il y a plusieurs difficultés qu'on ne peut expliquer, des profondeurs qu'on ne peut comprendre, des aventures qu'on ne peut croire, des prodiges qui révoltent la faible raison humaine, des contradictions qu'on ne peut concilier, c'est pour exercer notre foi, et pour humilier notre esprit ${ }^{71} »$, écrit-il dans l'article "Contradictions" des Questions sur l'Encyclopédie. Cette conception philosophique et éthique des antinomies propres à l'homme trouve son expression dans une esthétique du contraste, qui se caractérise par le recours récurrent aux oxymores («Mambrès à ce discours se sentit éclairé de quelques traits d'une lumière confuse qu'il ne démêlait pas encore ${ }^{72} »$ ) et aux zeugmas ( CCependant le taureau blanc tirant

67. Ibid., p. 83 .

68. A.-M. Garagnon, Cinq études sur le style de Voltaire, Orléans, Éditions Paradigme, 2008, p. 139 .

69. Le Taureau blanc, éd. citée, p. Io6.

70. «Le monde ne subsiste que de contradictions; que faudrait-il pour les abolir? Assembler les états du genre humain. Mais de la manière dont les hommes sont faits, ce serait une nouvelle contradiction s'ils étaient d'accord. Assemblez tous les lapins de l'univers, il n'y aura pas deux avis différents parmi eux." Article "Contradiction", Questions sur l'Encyclopédie, dans CEuvres complètes de Voltaire, 40, ouvr. cité, 2009, p. 236.

7I. Ibid., p. 266.

72. Le Taureau blanc, éd. citée, p. 7I. 
après lui sa chaîne et la vieille, était déjà parvenu auprès de la princesse qui était saisie d'étonnement et de peur $\left.{ }^{73} »\right)$. Ces figures ne sont pas seulement d'ordre stylistique : elles désignent la méthode du philosophe car c'est de la friction d'idées ou de motifs contradictoires que peut surgir la pensée.

La fin du conte est en ce sens riche de significations. À l'image du souper de Zadig qui réunit des hommes venus de tous les horizons pratiquant des religions diverses, le sacrement du taureau blanc en nouveau dieu Apis, sur lequel se conclut le conte, voit défiler les animaux vénérés comme des demi-dieux, "quarante mille guerriers", "un peuple innombrable», au son harmonieux du chœur des prêtres "qui élevait l'âme et l'attendrissait ${ }^{74}$. Significativement, le roi «surpris de ce spectacle» ne coupe pas le cou à sa fille : le défilé de ces figures symboliques a donc bel et bien un effet esthétique et émotionnel, grâce justement à l'harmonie de leurs contrastes. La fin du Taureau blanc se présente ainsi comme une expérience intersubjective, qui se rapproche de la conclusion de l'Histoire de Jenni. Dans ce conte, ce ne sont pas les discours de Freind qui parviennent à détourner Jenni de l'athéisme, c'est surtout le spectacle merveilleux de la voûte étoilée ${ }^{75}$ : comme l'a montré Colas Duflo, "[1]a scène, ici, fait philosophie» car elle rapporte "une authentique expérience existentielle ${ }^{76}$. Sans aller peut-être jusque-là, l'écriture poétique du Taureau blanc parvient bien à rassembler les temps et les espaces, à réunir les contraires : comme le dit René Pomeau, "[i]ci la thèse est dans le style ${ }^{77}$ ». Mais de nouveau, l'écriture voltairienne renverse ses propres propositions : les énumérations, notamment des fleurs et des instruments de musique («[...] on entendait résonner les sistres, les castagnettes, les tambours de basque, les psaltérions, les cornemuses, les harpes et les sambuques»), qui mêlent les odeurs et les époques différentes, peuvent se lire également comme une parodie du style biblique et confèrent au passage une tonalité légèrement bouffonne.

À l'instar des métamorphoses de Nabucodonosor, Le Taureau blanc se caractérise donc par une écriture anamorphosique. Lorsque Mambrès reconnaît la relativité de la portée de la Révélation, il conclut ainsi :

73. Ibid., p. 69.

74. Ibid., p. I3I.

75. «Parouba se mit à genoux et dit: "Les cieux annoncent Dieu". Tout l'équipage était autour du vénérable Freind, regardait et admirait.» Histoire de Jenni ou le Sage et l'Athée, ouvr. cité, p. II3.

76. C. Duflo, art. cité, p. 2 I.

77. R. Pomeau, art. cité, p. 26. 
Les merveilles que vous et les vôtres, et moi et les miens nous avons opérées, seront un jour un grand sujet de doute et de scandale pour les faux sages. Heureusement elles trouveront croyance chez les sages véritables qui seront soumis aux voyants dans une petite partie du monde, et c'est tout ce qu'il faut ${ }^{78}$.

Alors que les futurs suggèrent l'avènement du règne de la raison, capable de détruire les superstitions et le merveilleux (notamment chrétien), une opposition persiste entre les "faux sages" (les philosophes sceptiques) et "les sages véritables", c'est-à-dire les croyants soumis aux "voyants" (les prophètes $\left.{ }^{79}\right)$. Mais le propos peut être renversé : les "merveilles» ne sont-elles pas également celles du conte lui-même et son pouvoir d'éclairement des consciences, que peut-être certains mettront en doute? Dès lors, les «voyants» ne désigneraient-ils pas les philosophes conscients de leurs limites qui, à l'instar de Candide et de ses comparses, sont rassemblés «dans une petite partie du monde» pour «cultiver notre jardin »? La réversibilité interprétative du passage prouve ainsi l'impossibilité d'enfermer le conte dans une lecture univoque.

Invitant le lecteur à réfléchir par lui-même, Le Taureau blanc a donc bien une visée "morale», dans le sens que prend ce terme au XVIII ${ }^{\mathrm{e}}$ siècle. Pour les Lumières, morale et philosophie sont en effet quasiment synonymes car la moralité d'une action doit se libérer de toute transcendance et doit trouver son fondement dans la conscience humaine, dans la vérité trouvée grâce au travail critique ${ }^{80}$. La morale est désormais entendue comme la pratique d'un jugement autonome, qui repose sur la raison, comme le souligne Voltaire lui-même : "On ne peut trop répéter que tous les dogmes sont différents, et que la morale est la même chez tous les hommes qui font usage de leur raison. La morale vient donc de Dieu comme la lumière. Nos superstitions ne sont que ténèbres. Lecteur, réfléchissez: étendez cette vérité; tirez vos conséquences ${ }^{81}$. " Le conte philosophique, que l'on se raconte dans les salons ou qu'on lit dans les périodiques, contribue assurément à l'expansion de cet esprit critique.

78. Le Taureau blanc, éd. citée, p. 75.

79. Dom Calmet, Dictionnaire, Article «Prophète» : «Les Hébreux dans les commencements, les appelaient Voyants.» R. Pomeau, ouvr. cité, note 7, p. 75.

80. Dans son Essai sur l'entendement humain, Locke est sans doute le premier à distinguer «la loi divine qui règle ce qui est péché ou devoir» (transmise par révélation), «la loi civile [qui] est la règle du crime et de l'innocence» (la loi de l'État) et la loi spécifiquement morale, c'est-à-dire "la loi philosophique [qui] est la mesure du vice et de la vertu». J. Locke, Essai sur l'entendement humain, liv. II, chap. 28, \$ 7-II, P. Hamon (éd.), trad. P. Coste, Paris, Librairie générale, 2009, p. 545-549.

8I. Voltaire, Dictionnaire philosophique, ouvr. cité, p. 308. 
